\title{
Spin-Photon Entangling Diode
}

\section{Citation}

Flindt, Christian, Anders S. Sørensen, Mikhail D. Lukin, and Jacob M. Taylor. 2007. “SpinPhoton Entangling Diode." Physical Review Letters 98 (24). https://doi.org/10.1103/ physrevlett.98.240501.

\section{Permanent link}

http://nrs.harvard.edu/urn-3:HUL.InstRepos:41467467

\section{Terms of Use}

This article was downloaded from Harvard University's DASH repository, and is made available under the terms and conditions applicable to Other Posted Material, as set forth at http:// nrs.harvard.edu/urn-3:HUL.InstRepos:dash.current.terms-of-use\#LAA

\section{Share Your Story}

The Harvard community has made this article openly available.

Please share how this access benefits you. Submit a story.

\section{Accessibility}




\title{
Spin-Photon Entangling Diode
}

\author{
Christian Flindt, ${ }^{1,2,3}$ Anders S. Sørensen, ${ }^{2}$ Mikhail D. Lukin, ${ }^{3}$ and Jacob M. Taylor ${ }^{3,4}$ \\ ${ }^{1}$ MIC_Department of Micro and Nanotechnology, Technical University of Denmark, Kongens Lyngby 2800, Denmark \\ ${ }^{2}$ QUANTOP and the Niels Bohr Institute, University of Copenhagen, Copenhagen 2100, Denmark \\ ${ }^{3}$ Department of Physics, Harvard University, Massachusetts 02138, USA \\ ${ }^{4}$ Department of Physics, Massachusetts Institute of Technology, Massachusetts 02139, USA
}

(Received 28 February 2007; published 14 June 2007)

\begin{abstract}
We propose a semiconductor device that can electrically generate entangled electron spin-photon states, providing a building block for entanglement of distant spins. The device consists of a $p-i-n$ diode structure that incorporates a coupled double quantum dot. We show that electronic control of the diode bias and local gating allow for the generation of single photons that are entangled with a robust quantum memory based on the electron spins. Practical performance of this approach to controlled spin-photon entanglement is analyzed.
\end{abstract}

DOI: 10.1103/PhysRevLett.98.240501

Many practical approaches to quantum communication and computation rely upon interfacing stable quantum systems, which provide a good quantum memory, with carriers of quantum information (optical photons) at the level of single quanta. One promising approach to quantum memory uses electron spins confined in semiconductor quantum dots [1]. Quantum dots in diode structures can also be used for creating devices with novel electronic and optical properties. In particular, the Coulomb blockade exhibited by quantum dots is being used in experiments involving single charge and spin transport and manipulation [2-4] as well as for optical experiments such as generation of single-photons [5-8]. Application of these systems for quantum communication and computation protocols is a vibrant area of research [9-16].

In this Letter, we propose and analyze a novel semiconductor device in which an electrically pumped diode structure can combine controlled photonic interface with stable quantum memory. Such a device features purely electrical control over photonic and spin degrees of freedom. Specifically, we show that it can be used for controlled generation of entangled states between the frequency of an outgoing photon and the spin state of the electrons in a double quantum dot in the insulating layer of the diode similar to recent laser-driven experiments in atomic systems [17-19]. Using recently demonstrated techniques [20], the double-dot spin states can provide a robust quantum memory for long-term information storage, while outgoing photons can be used for probabilistic generation of long-distance entanglement in direct analogy to approaches being explored for atomic systems [21]. Finally, when integrated with gate-controlled quantum dot systems [22], this device could also form a building block for scalable quantum computation.

The basic idea of our approach can be understood by considering the semiconductor nanowire shown in Fig. 1(a), in which a Coulomb-blockade double quantum dot is sandwiched between the positively and negatively doped semiconductor regions, forming a $p-i-n$ diode. By
PACS numbers: 03.67.Mn, 71.35.-y, 73.40.Ty

manipulating the bias across the diode and the local gate, we can control the injection of electrons and holes into the double dot at the level of single charges. This allows us to electrically prepare a metastable exciton complex that a)

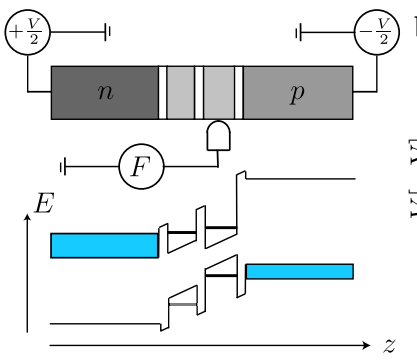

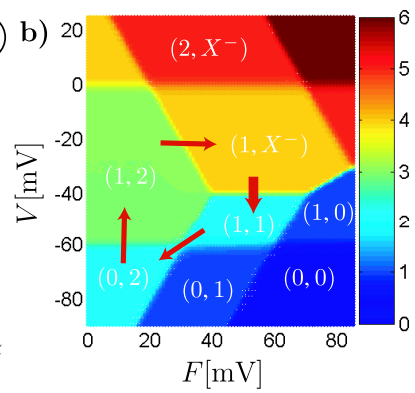

c)

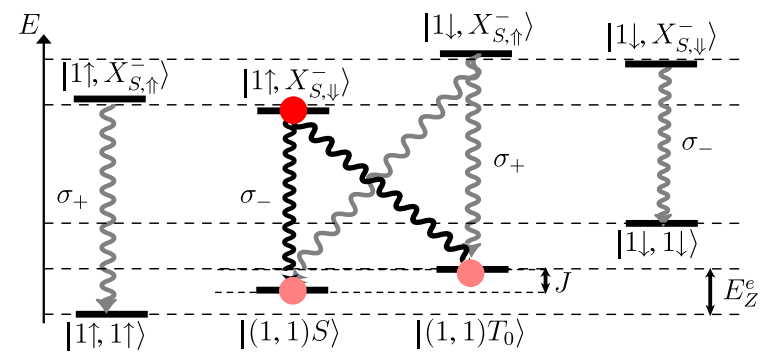

FIG. 1 (color online). Diode structure, charge stability diagram, and decay paths. (a) The device consists of a double quantum dot within the intrinsic region of a $p-i$ - $n$ diode structure. A schematic band-edge diagram is shown below. The left hole state (gray) is assumed to be energetically out of reach. (b) The stable charge configuration of the double quantum dot as a function of the bias $V$ and the local gate $F$. The scale bar indicates the total number of charges, while the labeling $(n, m)$ corresponds to the number of electrons on the left $(n)$ and right ( $m$ ) dot, replaced by $X^{-}$for dots containing a negatively charged exciton consisting of two electrons and a hole. As discussed in the text, the charging sequence indicated by arrows preferentially initializes the dots in the state $\left|1 \uparrow, X_{S, \Downarrow}^{-}\right\rangle$. (c) Initial and final states for excitonic recombination together with the polarization of the emitted photon. The desired decay processes (shown in black) are selected by filtering $\sigma_{-}$polarization. 
decays by electron-hole recombination to a charge configuration with a single electron in each of the two dots. When the two-electron spin states are split by the exchange coupling [1], the left circularly polarized photon that is emitted under the electron-hole recombination process will be frequency-entangled with the spin state of the remaining electrons [Fig. 1(c)].

Double dots can be grown inside $p-i-n$ junctions with techniques similar to those recently used to fabricate single quantum dot nanowire LEDs [23,24]. Alternatively, selfassembled dots on wafers can be used [25]. As illustrated in Fig. 1(a), the chemical potentials of the $p$ and $n$ regions on each side of the intrinsic region can be controlled by applying a bias across the device, while a gate electrode nearby the double dot can be used to tune the levels inside the dots independently of the applied bias. In what follows, we focus on III-V semiconductors, but in practice, other optically active materials can be used.

The electrostatic properties of the device can be visualized by using a charge stability diagram [26]. We describe the charge degrees of freedom of the double quantum dot using the Hamiltonian

$$
\hat{H}_{\mathrm{DQD}}=\hat{H}_{e e}^{l}+\hat{H}_{e e}^{r}+\hat{H}_{h h}^{r}+\hat{H}_{e h}^{r}+\hat{H}_{\tau}+\hat{H}_{F},
$$

where the Coulomb repulsion between similar charges $(q=e, h)$ on the left or right dot reads $\hat{H}_{q q}^{s}=U_{q q} \hat{n}_{q}^{s}\left(\hat{n}_{q}^{s}-\right.$ 1) $/ 2, s=l, r$, while the Coulomb attraction between electrons and hole in the right dot reads $\hat{H}_{e h}^{r}=-U_{e h} \hat{n}_{e}^{r} \hat{n}_{h}^{r}$. Here, $\hat{n}_{e(h)}^{\{r\}}$ is the operator for the number of electrons (holes) in the left \{right $\}$ dot. Tunneling of electrons between the dots with tunnel coupling $\tau$ is contained in the term $\hat{H}_{\tau}$, while $\hat{H}_{F}=-e F\left(\hat{n}_{h}^{r}-\hat{n}_{e}^{r}\right)$ incorporates the shift of the electron and hole states in the right dot due to the local gate $F$. Electrons with spin $\sigma= \pm 1 / 2$ on the left and right dots are created by $\hat{c}_{l, \sigma}^{\dagger}$ and $\hat{c}_{r, \sigma}^{\dagger}$, respectively, while heavy holes in the right dot are described by $\hat{d}_{r, \Sigma}^{\dagger}$, with $\Sigma= \pm 3 / 2$. In order for the scheme we consider to work ideally, we assume in the following that the hole states of the left dot remain unoccupied. This could occur due to the substantial band gap differences $(0.1 \mathrm{eV}$ or more $)$ between the two dots, due, e.g., to strain and dot size differences. Electrons are injected into the left dot from the electron Fermi sea in the $n$-region at chemical potential $\mu_{n}$, while holes are injected into the right dot from the hole Fermi sea in the $p$-region at chemical potential $\mu_{p}$. Assuming weak coupling to the electron and hole Fermi seas, we solve numerically the master equation for the probability of occupying the different many-body eigenstates of the double dot and find the stable charge configurations for the chosen parameter range.

In Fig. 1(b), we show the resulting charge stability diagram, where the total number of charges on the double quantum dot is given as function of the bias across the device $V$ and the local gate $F$. Here, $\mu_{n}=\mu_{n}^{0}+e V / 2$ and $\mu_{p}=\mu_{p}^{0}-e V / 2$, where $\mu_{n}^{0}$ and $\mu_{p}^{0}$, given by the doping levels of the $n$ and $p$ regions, respectively, determine the filling of the double dot without applied voltages. In the numerical calculations, the values of $\mu_{n}^{0}$ and $\mu_{p}^{0}$ were used to fix the occupations of the dots at $V, F=0$. For the shown charge stability diagram we have used the parameters $U_{e e} \simeq 30 \mathrm{meV}, U_{h h} \simeq 50 \mathrm{meV}, U_{e h} \simeq 40 \mathrm{meV}, \tau=$ $1 \mathrm{meV}$, and the tunnel couplings to the electron and hole Fermi seas being identical and much smaller than the temperature $T=4 \mathrm{~K}$. With a given setting of $V$ and $F$, the system rapidly reaches the corresponding stable charge configuration. On the figure, we also indicate the charge configuration of each of the two dots, where the labeling $(n, m)$ refers to the charges on the left $\operatorname{dot}(n)$ and the right $\operatorname{dot}(m)$, respectively. For configurations with no holes, the two labels correspond to the number of electrons in the left and right dot, respectively, while the symbol $X^{-}$denotes a negatively charged exciton consisting of two electrons and a hole. Such excitonic states have previously successfully been generated and controlled optically [27,28], but the procedure presented here works all electrically and thereby does not require any laser control. An external magnetic field $B$ is applied to the system parallel to the light emission and growth axis [the $z$-axis on Fig. 1(a)], i.e., in a Faraday configuration. In order to have reliable electron spin state preparation, we will require the Fermi seas to be sufficiently cold: $k_{B} T \ll\left|g^{*} \mu_{B} B\right|$ (see below).

We now describe the charge injection sequence indicated by arrows in Fig. 1(b). The sequence allows us repeatedly to prepare a desired spin and charge configuration, by injecting charges one at a time, and to direct its decay and corresponding photon emission process. We assume that single charges may be injected faster than the spontaneous decay time $(\sim 1 \mathrm{~ns}$ for GaAs selfassembled dots). By controlling the bias $V$ and the local gate $F$, the system is first put in the charge configuration ( 0 , 2 ). The expected ground state spin configuration of this state is a singlet due to the tight confinement of the electrons to a single dot: $|0,2 S\rangle=\hat{c}_{r, \uparrow}^{\dagger} \hat{c}_{r, \downarrow}^{\dagger}|0\rangle$. By increasing the bias, an additional spin- $\uparrow$ electron is added to the left dot, taking $|0,2 S\rangle$ to $|1 \uparrow, 2 S\rangle=\hat{c}_{l, \uparrow}^{\dagger} \hat{c}_{r, \uparrow}^{\dagger} \hat{c}_{r, \downarrow}^{\dagger}|0\rangle$. A spin- $\Downarrow$ heavy hole is now added to the right dot by control of the local gate, yielding the state $\left|1 \uparrow, X_{S, \Downarrow}^{-}\right\rangle=\hat{d}_{r, \Downarrow}^{\dagger} \hat{c}_{l, \uparrow}^{\dagger} \hat{c}_{r, \uparrow}^{\dagger} \hat{c}_{r, \downarrow}^{\dagger}|0\rangle$, which we expect to decay to $|1 \uparrow, 1 \downarrow\rangle=\hat{c}_{l, \uparrow}^{\dagger} \hat{c}_{r, \downarrow}^{\dagger}|0\rangle$ via excitonic recombination $\left(\hat{d}_{r, \Downarrow} \hat{c}_{r, \uparrow}\right)$. However, before recombination takes place, we rapidly (faster than the decay rate) move to the region, where $(1,1)$ is the stable charge configuration, hereby preventing emission of more than one photon (by refilling of an electron and a hole) in each cycle of the sequence.

The exciton decay couples the state $\left|1 \uparrow, X_{S, \Downarrow}^{-}\right\rangle$to $|1 \uparrow, 1 \downarrow\rangle$. With finite tunnel coupling between the left and right dots, this may be written as a superposition $|1 \uparrow, 1 \downarrow\rangle=$ $\left[|(1,1) S\rangle+\left|(1,1) T_{0}\right\rangle\right] / \sqrt{2}$, of the exchange-split singlet and triplet eigenstates $\left|(1,1) S\left(T_{0}\right)\right\rangle=2^{-1 / 2}\left(\hat{c}_{l, \uparrow}^{\dagger} \hat{c}_{r, \downarrow}^{\dagger} \pm\right.$ $\left.\hat{c}_{l, l}^{\dagger} \hat{c}_{r, \uparrow}^{\dagger}\right)|0\rangle$. Since $S$ and $T_{0}$ have different energies, the 
frequency of the outgoing photon will be entangled with the spin state [see Fig. 1(c) and Eq. (2) below]. These $S-T_{0}$ spin states were used in recent double-dot experiments where it was shown that they form a decoherence free subspace when manipulated with fast spin-echo pulses $[3,29,30]$. With the system in Faraday configuration, the spin of the hole determines the polarization of the emitted photon. While a spin- $\Downarrow$ heavy-hole recombines with a spin- $\uparrow$ electron under emission of a left-hand circularly polarized $\left(\sigma_{-}\right)$photon, a spin- $\Uparrow$ heavy-hole recombines with a spin- $\downarrow$ electron under emission of a right-hand circularly polarized $\left(\sigma_{+}\right)$photon. With temperature being comparable to or larger than the (small) hole Zeeman energy, the injected hole has a random spin state. With suitable polarization filtering, it is, however, possible to exclude photons that have been emitted with the heavyhole incorrectly in the spin- $\Uparrow$ state; thus, in practice when the hole $g$-factor is substantially smaller than the electronic $g$-factor or has the opposite sign, the possibility of incorrect hole spin states does not limit our approach. However, we still require $k_{b} T \ll\left|g^{*} \mu_{B} B\right|$ for the reliable preparation of the desired electron spin state in the left dot as this electron spin has no effect on the final polarization of the photon.

The resulting spin-photon entangled state can be used for generating spin-spin entanglement between two remote devices by interfering the emitted photons on a beam splitter as shown in Fig. 2(a) [31]. If the spin state in both devices are identical, both incoming photons can be mode matched in space, frequency, and time, so that HongOu-Mandel bunching will occur, leading to photon detection in only one arm of the beam splitter. On the other hand, if the spin states are different, the photons are distinguishable, and no "bunching" will occur. A photon detection in each arm of the beam splitter therefore leads to an entangled state of the spins in the spatially separated devices $\left|\Psi_{12}\right\rangle=\left(|S\rangle_{1}\left|T_{0}\right\rangle_{2}-\left|T_{0}\right\rangle_{1}|S\rangle_{2}\right) / \sqrt{2}$, where we have omit-
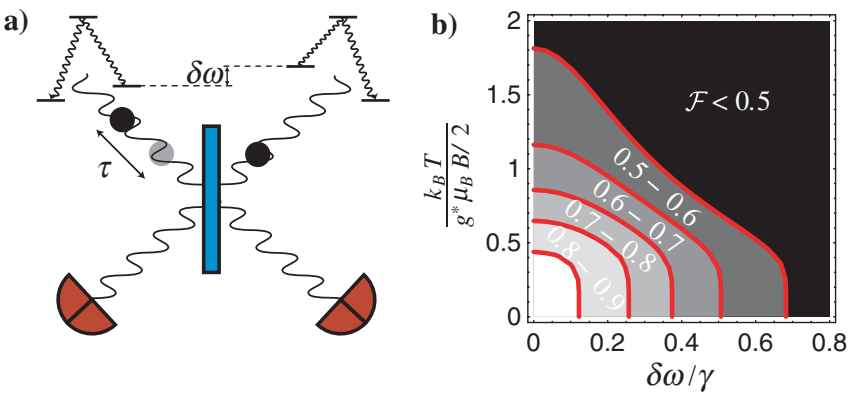

FIG. 2 (color online). Beam splitter setup and entanglement fidelity. (a) Photon interference leading to entanglement between two devices. The entanglement fidelity may be reduced due to different arrival times $\tau$ at the beam splitter, mismatch $\delta \omega$ between energy splittings in the two devices, and incorrect spin initialization (not indicated). (b) Entanglement fidelity $\mathcal{F}$ as function of temperature $T$ and energy mismatch $\delta \omega$. For the calculations, we have used $\gamma \times \bar{\tau}=0.03$. In the white region, $\mathcal{F}>0.9$. ted the charge labeling $(1,1)$. In the following, we consider the distinguishability of our outgoing photons to determine the requirements for such entanglement generation.

We first consider spontaneous decay associated with electron-hole recombination in a single device. The process can be described within the framework of WignerWeisskopf theory yielding a characteristic decay rate $\gamma$. We note that the ground state charge configuration $(1,1)$ may also be reached by the electron-hole pair tunneling back into the Fermi seas with rate $\Gamma_{o}$ rather than recombining. This does not impact the fidelity of entanglement, since no photon is emitted and we always condition on two clicks in the detectors, but it does reduce the success probability. After spontaneous decay has taken place the combined state $|\Phi\rangle$ (conditioned on electron-hole recombination) of the charges and the photon field reads

$$
|\Phi\rangle=\frac{1}{\sqrt{2}}\left[|S\rangle \otimes \hat{\xi}^{\dagger}\left(\omega_{S}\right)|0\rangle+\left|T_{0}\right\rangle \otimes \hat{\xi}^{\dagger}\left(\omega_{T_{0}}\right)|0\rangle\right],
$$

where $\quad \hat{\xi}^{\dagger}(\omega)=\sum_{k} \xi(\omega, k) \hat{a}_{k}^{\dagger} \quad$ with $\quad \xi(\omega, k)=\frac{1}{\sqrt{2 \pi}} \times$ $\frac{\sqrt{\gamma} e^{-i k z_{0}}}{\left(\omega_{k}-\omega\right)+i \gamma / 2}$ and $\hat{a}_{k}^{\dagger}$ being the creation operator for photons of mode $k$. Here, $|0\rangle$ is the vacuum state of the photon field, while the position of the double quantum dot is $z_{0}$, and $\omega_{S}$ and $\omega_{T_{0}}$ denote the splittings between the excited state and the singlet and triplet ground states, respectively, so that $\left|\omega_{S}-\omega_{T_{0}}\right|$ equals the exchange coupling $J$. The width of the photon wave packet is given by $\gamma=\gamma_{S}+\gamma_{T_{0}}+\Gamma_{o}$, and above we have taken the same rates, $\gamma_{S}=\gamma_{T_{0}}$, resulting in equal branching ratios for the two processes.

We now consider the beam splitter setup depicted in Fig. 2(a) and consider two photons emitted by similar devices. With probability $1 / 2$, the two photons are in states corresponding to the same spin state of the electrons in the two devices (both singlet or both triplet). The probability of detecting two photons, in states $\left|\Psi_{L}\right\rangle=\xi_{L}^{\dagger}\left(\omega_{L}\right)|0\rangle$ and $\left|\Psi_{R}\right\rangle=\xi_{R}^{\dagger}\left(\omega_{R}\right)|0\rangle$, respectively, at different detectors (denoted $L$ and $R$ ) after they have scattered on the 50/50 beam splitter is $P\left(1_{L}, 1_{R}\right)=\left(1-|\mathcal{J}|^{2}\right) / 2$, where $\mathcal{J} \equiv$ $\sum_{k} \xi_{L}\left(\omega_{L}, k\right) \xi_{R}^{*}\left(\omega_{R}, k\right)$ is the overlap of the wave packet amplitudes. With $\omega_{L}=\omega_{S}$ and $\omega_{R}=\omega_{T_{0}}$, we find

$$
P\left(1_{L}, 1_{R}\right)=\frac{1}{2}\left(1-\frac{\gamma^{2}}{\gamma^{2}+J^{2}}\right) .
$$

Typical electron-hole recombination rates are on the order of $\mathrm{GHz}$, and the width of the wave packets $\gamma$ therefore is on the order of $\mu \mathrm{eVs}$, which is smaller than the exchange coupling $J$ between tunnel-coupled quantum dots, which is in the range $10 \mu \mathrm{eV}$ to $1 \mathrm{meV}$. Thus, we expect $P\left(1_{L}, 1_{R}\right) \sim 1 / 2$ and a corresponding success rate of $\eta^{2} / 4$ for detecting the two photons at different detectors, where $\eta$ is the combined single photon emission and detection probability.

Besides the success rate, we need to consider the fidelity $\mathcal{F}=\left\langle\Psi_{12}\left|\hat{\rho}_{s}\right| \Psi_{12}\right\rangle$ of the entangling procedure, where $\left|\Psi_{12}\right\rangle$ is the desired state, and $\hat{\rho}_{s}$ is the reduced density 
matrix for the spins in the two devices. Possible error processes include wrong initialization of the spin states, jitter in the hole injection and path length differences leading to different arrival times at the beam splitter, and different energy splittings between the excited state and the ground states in the two devices. We remark that the specific device details could be tuned, e.g., with dc Stark shifts, to minimize these errors. To evaluate the effect of jitter and the different energy splittings, we write the full density matrix of the spins and photons as $\hat{\rho}=$ $|\Phi\rangle_{1}|\tilde{\Phi}\rangle_{22}\left\langle\left.\tilde{\Phi}\right|_{1}\langle\Phi| \text { where both } \mid \Phi\right\rangle_{1}$ and $|\tilde{\Phi}\rangle_{2}$ are of the form given in Eq. (2), but $|\tilde{\Phi}\rangle_{2}$ has a component on a field mode perpendicular to the field mode emitted by device number 1 ; i.e., for $|\tilde{\Phi}\rangle_{2}, \quad \hat{\xi}^{\dagger}$ is replaced by $\mathcal{J} \hat{\xi}^{\dagger}+$ $\sqrt{1-|\mathcal{J}|^{2}} \hat{\xi}_{\text {err }}^{\dagger}$. Here, $\hat{\xi}_{\text {err }}^{\dagger}$ creates a photon in an undesired mode due to the jitter and energy shifts, and $\mathcal{J}$ denotes the corresponding wave packet overlap. Conditioned on clicks in different detectors after the beam splitter, we find that the erroneous field component generates the spin states $\left|T_{0}\right\rangle_{1}\left|T_{0}\right\rangle_{2},|S\rangle_{1}|S\rangle_{2},\left|T_{0}\right\rangle_{1}|S\rangle_{2}$, and $|S\rangle_{1}\left|T_{0}\right\rangle_{2}$ with equal probability $\left(1-|\mathcal{J}|^{2}\right) /\left(4-3|\mathcal{J}|^{2}\right)$. The fidelities corresponding to these states are $0,0,1 / 2$, and $1 / 2$, respectively. The desired state $\left(|S\rangle_{1}\left|T_{0}\right\rangle_{2}-\left|T_{0}\right\rangle_{1}|S\rangle_{2}\right) / \sqrt{2}$ (with fidelity 1) is generated with probability $|\mathcal{J}|^{2} /\left(4-3|\mathcal{J}|^{2}\right)$. Combining these numbers, we find the fidelity $1 /(4-$ $3|\mathcal{J}|^{2}$ ), which, however, does not yet include the possibility of wrong spin initialization.

In thermal equilibrium, the probability of initializing the wrong spin state $|\downarrow\rangle$ in the left dot is given by the Boltzmann factor $p_{\downarrow} \propto e^{-g^{*} \mu_{B} B / 2 k_{B} T}$. Wrong initialization of the spin in one or both of the devices leads to states with fidelity 0 . For the probability of detection at different detectors due to a wrong spin in one or both of the devices, we use the upper bound $\eta^{2}\left(2 p_{\downarrow}-p_{\downarrow}^{2}\right) / 2$. Including this estimate for the effect of wrong spin initialization in the above expression for the fidelity, we find

$$
\mathcal{F}=\frac{1}{4-3|\mathcal{J}|^{2}} \frac{1}{1+2\left(2 p_{\downarrow}-p_{\downarrow}^{2}\right)} .
$$

Two photons created by $\hat{\xi}^{\dagger}(\omega)$ and $\hat{\xi}^{\dagger}(\omega+\delta \omega)$ with a time difference $\tau$ have the wave packet overlap $|\mathcal{J}|^{2}=$ $\frac{\gamma^{2} e^{-\gamma|\tau|}}{\delta \omega^{2}+\gamma^{2}}$. For the time difference, we assume a Gaussian probability distribution with width $\bar{\tau}, \mathcal{P}(\tau) \propto e^{-(\tau / \bar{\tau})^{2} / 2}$. This distribution is relevant when noise in the gates controlling the hole injection is responsible for the photons being created at different times or when the optical paths do not have exactly the same length. When evaluating the fidelity, we average the expression in Eq. (4) with respect to the Gaussian distribution. In Fig. 2(b), we show the fidelity as function of temperature and energy mismatch. We see that with realistic parameters, it is possible to obtain a high degree of fidelity, $\mathcal{F}>0.9$, and even with temperatures comparable to the Zeeman energy, the fidelity may be larger than 0.5 , the lower bound for the use of entanglement purification protocols [32]. Furthermore, the loss of fidelity due to time jitter or energy mismatch may be suppressed by gating the detectors in time, thereby improving the shown results.

In conclusion, we have presented a proposal for an allelectrically controlled device for long-range electron spin entanglement and shown that entanglement can be generated with a high degree of fidelity using available experimental techniques. When combined with existing quantum optical methods and solid-state technologies for spin manipulation and detection, our proposed device may form an important building block in future quantum communication and information processing architectures.

We thank M. V. G. Dutt, K. Flensberg, A. Imamoglu, and M. Stopa for helpful conversations. The work was supported by the Denmark-America Foundation, Pappalardo, the Danish Natural Science Research Council, NSF, DTO, and the Packard Foundation.

[1] D. Loss and D.P. DiVincenzo, Phys. Rev. A 57, 120 (1998); G. Burkard, D. Loss, and D. P. DiVincenzo, Phys. Rev. B 59, 2070 (1999).

[2] M. Kroutvar et al., Nature (London) 432, 81 (2004).

[3] J. R. Petta et al., Science 309, 2180 (2005).

[4] F. H. L. Koppens et al., Nature (London) 442, 766 (2006).

[5] A. Imamoglu and Y. Yamamoto, Phys. Rev. Lett. 72, 210 (1994).

[6] J. Kim et al., Nature (London) 397, 500 (1999).

[7] P. Michler et al., Science 290, 2282 (2000).

[8] A. J. Bennett et al., Phys. Status Solidi B 243, 3730 (2006).

[9] G. Chen et al., Science 289, 1906 (2000).

[10] O. Gywat, G. Burkard, and D. Loss, Phys. Rev. B 65, 205329 (2002).

[11] W. Yao, R.-B. Liu, and L. J. Sham, Phys. Rev. Lett. 95, 030504 (2005).

[12] E. Waks et al., Nature (London) 420, 762 (2002).

[13] A. Badolato et al., Science 308, 1158 (2005).

[14] H. J. Krenner et al., Phys. Rev. Lett. 97, 076403 (2006).

[15] E. A. Stinaff et al., Science 311, 636 (2006).

[16] R. M. Stevenson et al., Nature (London) 439, 179 (2006).

[17] B. B. Blinov et al., Nature (London) 428, 153 (2004).

[18] J. Volz et al., Phys. Rev. Lett. 96, 030404 (2006).

[19] W. Rosenfeld et al., Phys. Rev. Lett. 98, 050504 (2007).

[20] J. M. Taylor et al., Phys. Rev. Lett. 94, 236803 (2005).

[21] L. M. Duan et al., Nature (London) 414, 413 (2001).

[22] H. A. Engel et al., arXiv:cond-mat/0612700.

[23] M. T. Borgstrom et al., Nano Lett. 5, 1439 (2005).

[24] E. D. Minot et al., Nano Lett. 7, 367 (2007).

[25] G. S. Solomon et al., Phys. Rev. Lett. 76, 952 (1996).

[26] W. G. van der Wiel et al., Rev. Mod. Phys. 75, 1 (2003).

[27] M. V. G. Dutt et al., Phys. Rev. Lett. 94, 227403 (2005).

[28] M. Atatüre et al., Science 312, 551 (2006).

[29] J. Levy, Phys. Rev. Lett. 89, 147902 (2002).

[30] J. M. Taylor et al., Nature Phys. 1, 177 (2005).

[31] L. M. Duan et al., Phys. Rev. A 73, 062324 (2006).

[32] H. J. Briegel et al., Phys. Rev. Lett. 81, 5932 (1998). 